\title{
Response to rapid infusion or diuresis in acute cardiac infarction
}

\author{
D. J. Coltart and John Hamer \\ From Cardiac Department, St. Bartholomew's Hospital, London E.C.I
}

The effect on the right atrial pressure and oxygen saturation of expansion of the plasma volume by rapid infusion of $500 \mathrm{ml}$. 5 per cent glucose has been studied in I4 patients with recent uncomplicated cardiac infarction, and in 5 patients with cardiogenic shock. The findings are contrasted with the effect of intravenous frusemide ( $40 \mathrm{mg}$.) in 8 patients with recent cardiac infarction complicated by pulmonary congestion, as judged from the chest radiograph.

The right atrial pressure and oxygen saturation are a useful guide to the haemodynamic state of the patient, and can be obtained without undue risk or disturbance. There is a high correlation between the right atrial oxygen saturation and the coronary prognostic index on admission to hospital.

Rapid infusion produced a significant increase in right atrial oxygen saturation in the uncomplicated patients; there was no rise in right atrial pressure, and pulmonary congestion was not produced. Three of the 5 patients with shock showed improvement in right atrial oxygen saturation without a rise in right atrial pressure, and 2 recovered. Frusemide produced improvement in both right atrial pressure and oxygen saturation in the patients with pulmonary congestion.

The favourable response to a fluid load in some patients suggests that an increase in filling pressure is sometimes needed in cardiac infarction to obtain an optimal ventricular response. $A$ high venous pressure in patients without evidence of pulmonary congestion is not an indication for diuretic treatment.

Detailed observation in patients with acute cardiac infarction may detect changes in myocardial function at an early stage. Monitoring may range from serial clinical observation to left heart catheterization (Nixon, Taylor, and Morton, 1968; Cohn, Tristani, and Khatri, 1969). The added risk of more detailed studies may be thought justifiable in serious situations if the information obtained is helpful in the management of the patient. However, there is a need for a simpler way of assessing the changes in the circulation that can be applied to any patient with cardiac infarction with little risk or disturbance so that deterioration can be detected at an early stage.

We have made use of the simple measurements that can be obtained from the venous catheter which is often inserted in patients with cardiac infarction for therapeutic purposes. If the catheter is advanced to the right atrium, measurements of pressure and oxygen saturation give an indication of ventricuReceived 4 June 1970. lar filling pressure and cardiac output and provide a useful guide to changes in the haemodynamic situation.

We have used this technique to assess the response to a fluid load in uncomplicated or shocked patients, and have compared the findings to the response to a rapidly-acting diuretic in patients showing pulmonary congestion.

\footnotetext{
Methods

Patients with acute myocardial infarction were diagnosed on the basis of the characteristic clinical features of cardiac infarction, the presence of pathological $Q$ waves with evolutionary ST-T wave changes of a recent cardiac infarction in the electrocardiogram, and a rise in the serum aspartate aminotransferase level to over 40 units $/ \mathrm{ml}$. The severity of infarction was assessed on admission from the coronary prognostic index of Norris et al. (1969).

Thirty-nine patients were studied; I4 had uncomplicated myocardial infarction, 12 had evidence of pulmonary venous congestion as judged
} 
by an increase in size of the upper lobe vessels or the presence of interstitial or alveolar pulmonary oedema on chest $x$-ray at the time of admission, - and 5 patients were in cardiogenic shock, i.e. cold, sweaty, and oliguric with a systolic blood pressure persistently below $100 \mathrm{~mm}$. Hg. Eight other patients, included initially, developed an arrhythmia needing special treatment and were not studied further.

A catheter was inserted percutaneously through a medial antecubital or external jugular vein and

- positioned at a high right atrial level. The position was confirmed by radiography in all patients. Mean right atrial pressure was recorded with a Statham P23Db strain gauge with zero level at the sternal angle. Right atrial oxygen saturation was determined with $5 \mathrm{ml}$. of blood aspirated into a $5 \mathrm{ml}$. heparinized glass syringe after approximately 3-5 ml. blood had been removed to clear the

- line. The blood was rapidly transferred to a glass cuvette and oxygen saturation measured by the Kipp reflection oximeter. The oximeter was calibrated using standards supplied with the machine and checked against blood oxygen saturation determined by the van Slyke method. The reproducibility of measurements made with this instrument was found on duplicate determinations to be $\pm 2 \cdot 3$ per cent.

In the patients without evidence of pulmonary congestion, an infusion of $500 \mathrm{ml}$. of 5 per cent glucose was given into the catheter as rapidly as possible. The right atrial pressure was measured before and every 3 minutes after the infusion, for 60 minutes. If the pressure had risen more than $5 \mathrm{~mm}$. $\mathrm{Hg}$, the infusion was terminated and restarted if the pressure returned to the previous value within 9 minutes. Using this precaution the mean time of infusion was 13 minutes. Right atrial oxygen saturation was determined before and every ro minutes after the infusion, for 40 minutes. Five patients in this group were observed with serial pressure and oxygen saturation recordings every 15 minutes for one hour before infusion.

Patients with any evidence of pulmonary venous congestion on the admission chest $x$-ray were excluded from the infusion study. These patients had mean right atrial pressures and right atrial oxygen saturations taken before and one hour after $40 \mathrm{mg}$. frusemide intravenously. Eight of the patients subsequently to be given frusemide were observed for an hour before with pressure and oxygen saturation recordings every is minutes.

Arterial blood samples were obtained for Astrup measurements from 8 patients during the course of the study and the oxygen saturation was measured. Five of the samples were from the shocked patients and the other 3 measurements were in patients in heart failure.

Throughout this study oxygen was not administered. No patient was studied within an hour of receiving intravenous analgesics and all were at complete rest throughout the course of the study.

Statistical comparison between the different groups of patients was by an analysis of variance.

\section{Results (Tables I and 2)}

There is a good correlation between the right atrial oxygen saturation on admission and the coronary prognostic index (Fig. I). Of the 4 patients who died in the series, all had admission right atrial oxygen saturations below 50 per cent, and right atrial pressures above Io $\mathrm{mm}$. Hg. There was a significantly $(\mathrm{p}<0.00 \mathrm{r})$ lower right atrial oxygen saturation on admission in the patients with heart failure or shock. There was also a significantly $(p<0.01)$ greater admission right atrial pressure in these patients.

There was no significant change in the right atrial oxygen saturation in the period of pre-

TABLE I Right atrial oxygen saturations

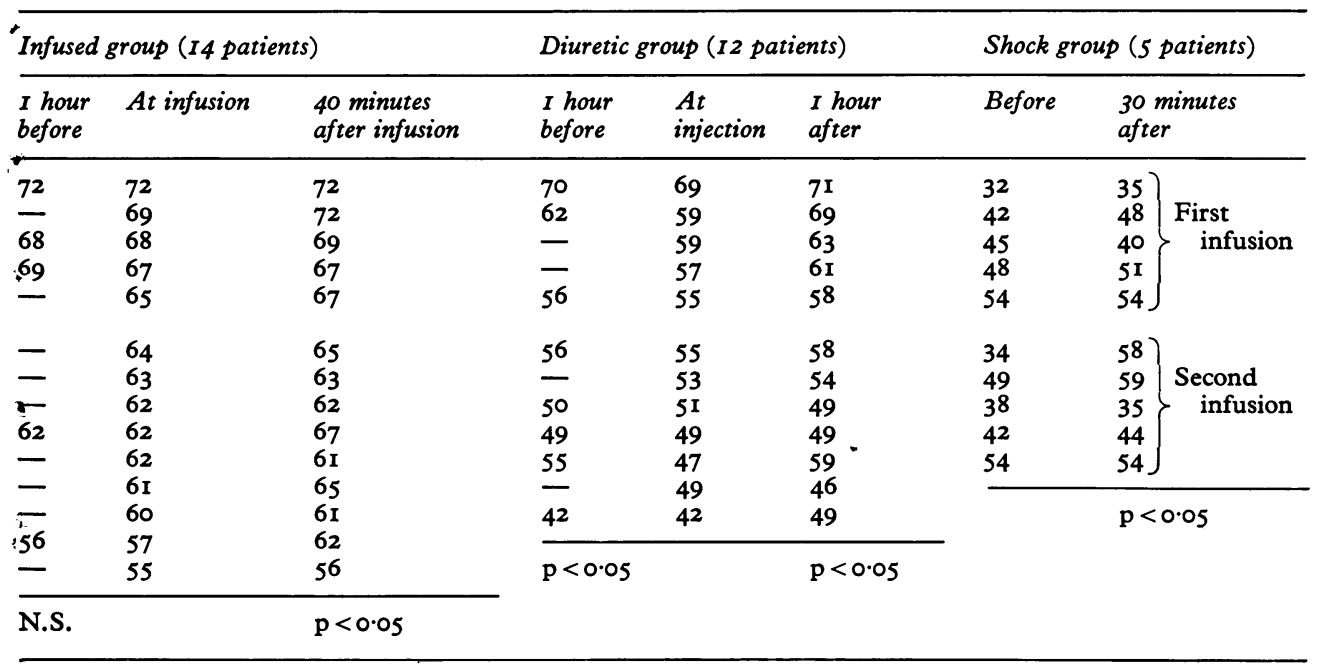


TABLE 2 Right atrial pressures

\begin{tabular}{|c|c|c|c|c|c|c|c|c|}
\hline \multicolumn{3}{|c|}{ Infused group ( 14 patients) } & \multicolumn{3}{|c|}{ Diuretic group ( 12 patients) } & \multicolumn{3}{|c|}{ Shock group ( 5 patients) } \\
\hline $\begin{array}{l}\text { I hour } \\
\text { before }\end{array}$ & At infusion & I hour after & $\begin{array}{l}\text { I hour } \\
\text { before }\end{array}$ & $\begin{array}{l}\text { At } \\
\text { injection }\end{array}$ & $\begin{array}{l}\text { I hour } \\
\text { after }\end{array}$ & $\begin{array}{l}\text { At } \\
\text { infusion }\end{array}$ & $\begin{array}{l}30 \mathrm{~m} \\
\text { after }\end{array}$ & ninutes \\
\hline \multirow{5}{*}{$\begin{array}{l}\frac{14}{10 \cdot 5} \\
10 \\
-\end{array}$} & 14 & 15 & 20 & 20 & I6 & I7 & 13? & \multirow{5}{*}{$\begin{array}{l}\text { First } \\
\text { infusion }\end{array}$} \\
\hline & II & 15 & I6 & 17 & 15 & II & 8 & \\
\hline & 10.5 & 10.5 & - & I5 & 12 & 2 & $10\}$ & \\
\hline & 10 & 10 & - & 13 & II & 14 & I4 & \\
\hline & 8 & 10 & I I & II & 9 & I0 & 10 & \\
\hline- & 8 & 7 & 12 & II & 9 & 13 & I0) & \multirow{5}{*}{$\begin{array}{l}\text { Second } \\
\text { infusion }\end{array}$} \\
\hline - & I I & 5 & - & I0 & 9 & 7 & 4 & \\
\hline- & 6 & 5 & 9 & 9 & 9 & 3 & 5 & \\
\hline 2 & 4 & 3 & II & I0 & 3 & 10 & 9 & \\
\hline- & 4 & 2 & 8 & 7 & 3 & 8 & 9] & \\
\hline \multirow{2}{*}{$\overline{-}$} & 3.5 & 5 & 一 & 6 & 2 & \multirow{3}{*}{\multicolumn{3}{|c|}{ N.S. }} \\
\hline & $2 \cdot 5$ & 3 & 2 & 2 & 2 & & & \\
\hline$\underline{2}$ & $\begin{array}{l}2 \\
1 \cdot 5\end{array}$ & $1 \cdot 5$ & N.S. & & $p<0.05$ & & & \\
\hline N.S. & & N.S. & & & & & & \\
\hline
\end{tabular}

liminary observation in the patients given an infusion. The saturation was significantly $(p<0.05)$ increased by infusion (Fig. 2). The right atrial pressure was not significantly altered by the control observations or after infusion (Fig. 3).

There was a significant $(p<0.05)$ decrease in right atrial oxygen saturation in the heart failure group before the diuretic. The saturation was significantly $(p<0.05)$ increased by the diuretic (Fig. 4). The right atrial pressure was unaltered during the control observations but was significantly $(p<0.05)$ reduced (Fig. 5) by the diuretic treatment.

FIG. I Shows degree of correlation between right atrial oxygen saturation and coronary prognostic index.

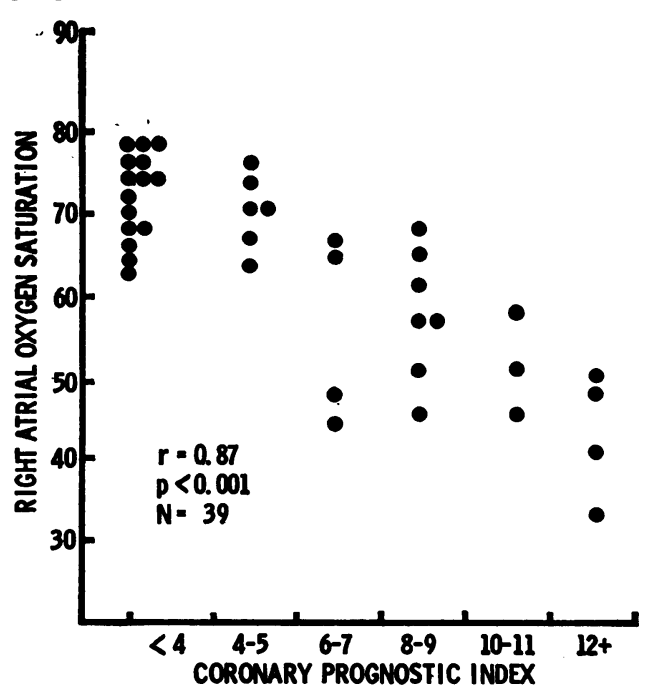

All 5 patients in shock were infused on two occasions with similar results each time. Three responded with an increase in the right atrial oxygen saturation (Fig. 6) and a decrease or no change in the right atrial pressure (Fig. 7). One patient (D) failed to maintain this improvement and at necropsy was found to have an extensively infarcted left ventricle. The other two patients have left hospital. In one patient (C) the right atrial pressure increased after infusion, with a decrease in right atrial oxygen saturation. This patient died as a result of ventricular fibrillation and at necropsy was found to have a large infarct. The patient (E) who did not have a seriously raised right atrial pressure on admission and did not re-

FIG. 2 Right atrial oxygen saturation in patients with no clinical or radiological evidence of heart failure before and after infusion.

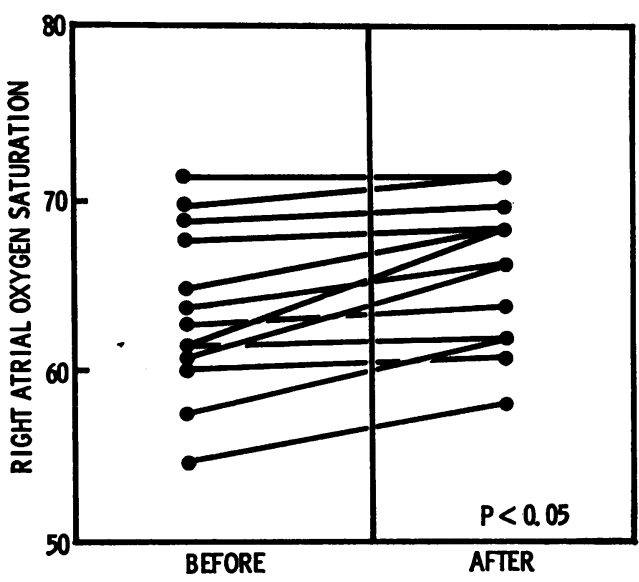




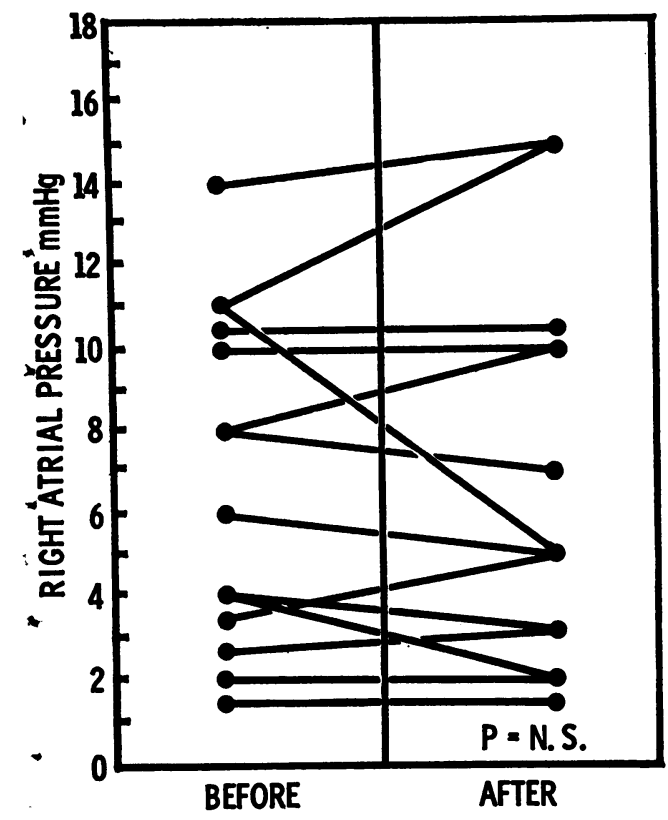

FIG. 3 Mean right atrial pressure in patients. with no clinical or radiological evidence of heart failure. Pressures standardized at the sternal angle.

spond to infusion was found at necropsy to have a relatively small infarct.

The arterial oxygen saturation was $93.2 \pm$ a. I per cent in the 8 patients studied. One episode of arrhythmia was probably related to the technique since atrial fibrillation reverted to sinus rhythm on withdrawal of the catheter. No radiological evidence of increased pulmonary congestion was encountered.

FIG. 4 Right atrial oxygen saturation in satients with heart failure before and after :reatment.

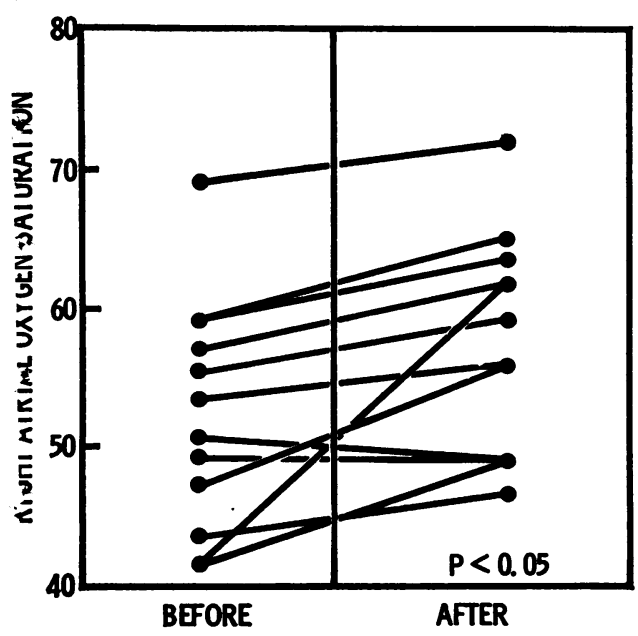

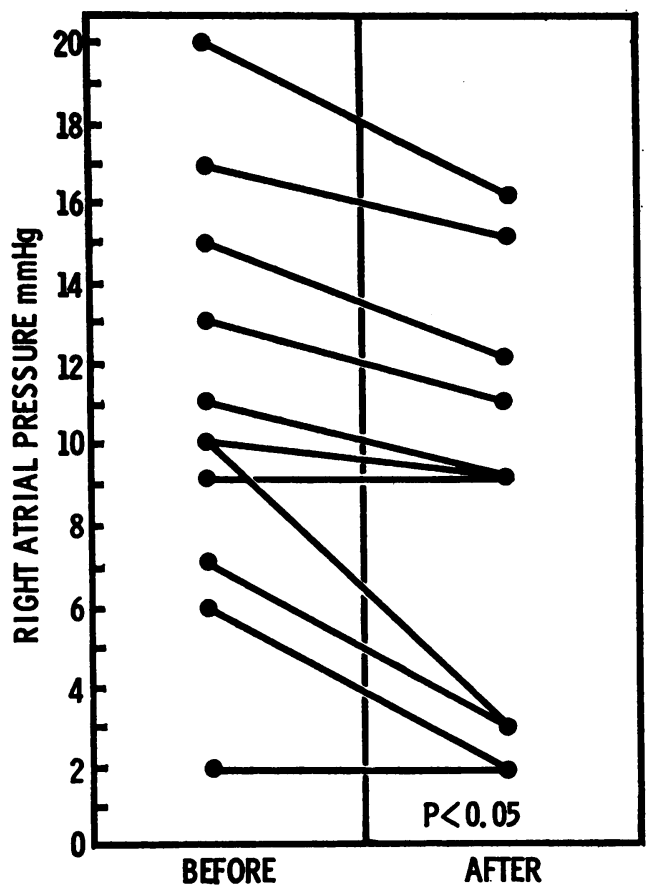

FIG. 5 Mean right atrial pressure in patients in heart failure before and after treatment. Pressures standardized to the sternal angle.

\section{Discussion}

The right atrial oxygen saturation is a good index of changes in the cardiac output when the arterial oxygen saturation and the oxygen consumption remain constant. The arterial oxygen saturation was not greatly reduced in the seriously ill patients whom we studied, but the effects of pulmonary changes on the

FIG. 6 Right atrial oxygen saturation in patients in shock before and after infusion.

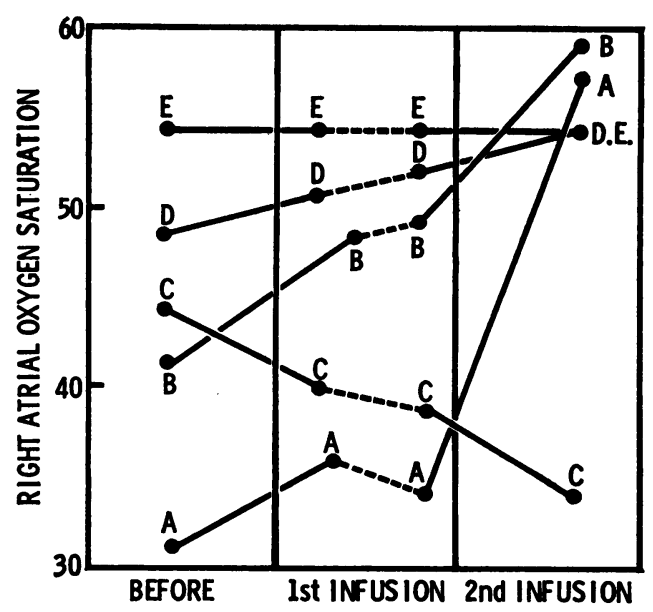




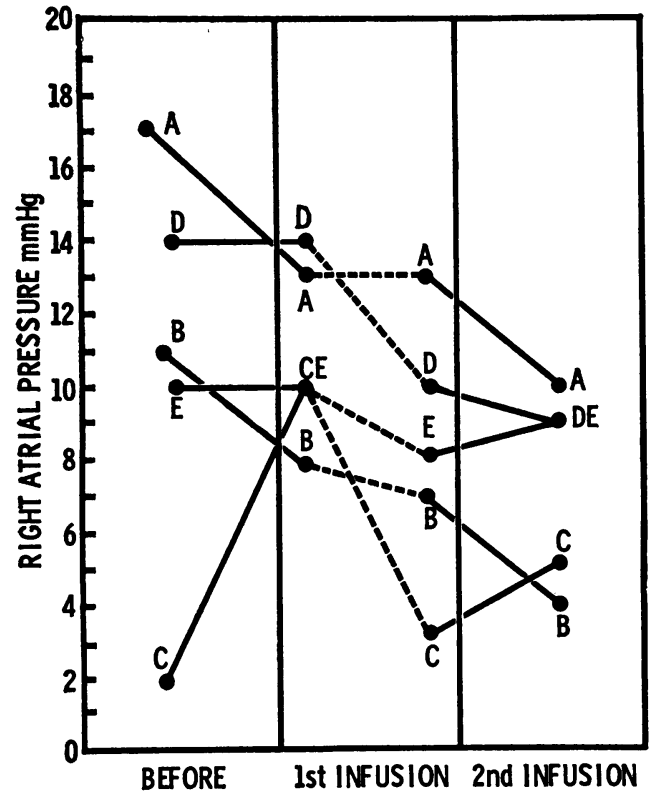

FIG. 7 Mean right atrial pressure in patients in shock before and after infusion. Pressures standardized at the sternal angle.

arterial oxygen saturation must be considered in the interpretation of our findings. The patients were kept completely at rest throughout the period of study, so little alteration in oxygen consumption would be expected.

Though pulmonary arterial samples are to be preferred as indicators of the mixed venous oxygen saturation, there is good evidence (Scheinman, Brown, and Rapaport, 1969) that right atrial samples give similar information, even in cardiac infarction with failure or shock. Superior vena caval samples, which have been used for this purpose, may be misleading because of the preferential maintenance of cerebral blood flow under these circumstances.

Hutter and Moss (1968) found that a right atrial oxygen saturation below 55 per cent was an indication of serious haemodynamic disturbance in patients with acute cardiac infarction. Our findings support this view, and the good correlation between the right atrial oxygen saturation on admission and the coronary prognostic index is an indication of the value of the measurement in the assessment of the outlook in the individual case. A fall in right atrial oxygen saturation may be produced by a reduction in the cardiac output or an increase in pulmonary congestion leading to a lower arterial saturation. In many cases both mechanisms will play a part, and the reduction of right atrial oxygen saturation is an early sign of deterioration.
As cardiac infarction is primarily a left ventricular disease, and the infarcted area tends to be stiffer than normal myocardium, the left ventricular filling pressure is of major interest in assessing the haemodynamic disturbance. However, the hazards of left heart catheterization make direct measurement of left ventricular or left atrial pressure undesirable except under special circumstances. The right atrial pressure is an indirect guide to the pressure changes in the left side of the heart (Cohn et al., 1969; Loeb et al., 1969). An increase in the volume of fluid in the circulation, and the effects of alterations in venous tone, will be evident on the right side of the heart as well as on the left. The right atrial pressure will generally be less raised than the left atrial pressure, as a greater filling pressure is needed to maintain the output of the diseased left ventricle. If the left atrial pressure rises substantially, however, an increase in pulmonary arterial pressure will put an additional load on the right ventricle and require a further increase in right atrial pressure.

In patients with shock after cardiac infarction, hypoxia or vascular reflexes may produce widespread peripheral vasodilatation and prevent the increase in filling pressure needed to maintain the best possible output from the damaged left ventricle. It has been suggested that under these circumstances a fluid load might have a beneficial effect on the circulation (Cohn et al., 1969; Allen, Danzig, and Swan, 1967; Nixon, Ikram, and Morton, 1967). Even if compensatory mechanisms are able to produce some increase in ventricular filling, evident as a raised right atrial pressure, the response may still be less than that needed for the best possible cardiac output.

A test infusion of fluid, as carried out in this study, is a useful guide to the need for expansion of circulating blood volume. A slow infusion of 5 per cent glucose is lost from the circulation before any haemodynamic effect can be evident, but a rapid infusion produces a definite but temporary increase in volume which can be maintained by further infusion of glucose or dextran if a sustained effect is needed. The frequent measurement of right atrial pressure and oxygen saturation allows termination of the infusion as soon as any deleterious effect is observed and minimizes the risk of producing serious pulmonary congestion.

The significant increase in right atrial oxygen saturation which we found after infusion in our patients without pulmonary congestion can be interpreted as indicating an increase in cardiac output. A response to the infusion seems likely as no change was found in the 
preliminary observation period. Right atrial pressure was not raised by the infusion, but if there had been any worsening of pulmonary congestion a fall in right atrial oxygen saturation secondary to a reduction in arterial oxygen saturation would have been expected. These findings suggest that the circulating blood volume is often below the optimal level in patients with cardiac infarction.

Three of our patients with shock showed improvement of the right atrial oxygen saturation after infusion, and right atrial pressure fell or was unchanged even though the initial pressure was raised. The poor prognosis in patients that do not respond to infusion has been noted by others (Allen et al., 1967). In one of our patients right atrial pressure rose and saturation fell, suggesting that the left ventricle could not respond to increased filling, and severe left ventricular damage was confirmed at necropsy. However, in the remaining patients the infarct was less extensive.

In our patients with radiological evidence of pulmonary congestion we measured the response to a rapidly-acting diuretic. Such treatment is often given under these circumstances, and may be essential to prevent fatal pulmonary oedema, but diuresis will not necessarily have a beneficial effect on left ventricular function which may require a high filling pressure. The right atrial oxygen saturation was falling before the diuretic in our patients, and increased afterwards with a corresponding fall in right atrial pressure. Our technique does not allow a distinction between the effect on arterial oxygen saturation of relief of pulmonary congestion or of an improvement in cardiac output following the diuresis. Either of these responses would lead to an increase in right atrial oxygen saturation. Fur,ther studies with arterial oxygen saturation measurements will be needed to clarify this point.

\section{-Conclusions}

Patients with acute cardiac infarction and a low right atrial pressure may have an inade- quate blood volume. Rapid infusion has a favourable effect in these patients and we suggest a wider trial of this treatment in acute cardiac infarction.

The procedure may be beneficial in patients with shock and may be used with caution when the right atrial pressure is raised.

In the absence of shock, pulmonary congestion or a high right atrial pressure suggests that infusion is not needed, but inappropriate diuretic therapy may be harmful as the damaged left ventricle needs a raised filling pressure.

We are grateful to the physicians of St. Bartholomew's Hospital for allowing us to study the patients under their care.

\section{References}

Allen, H. N., Danzig, R., and Swan, H. J. C. (1967). Incidence and significance of relative hypovolemia as a cause of shock associated with acute myocardial infarction. Circulation, 36, Suppl. II, 50.

Cohn, J. N., Tristani, F. E., and Khatri, I. M. (1969). Studies in clinical shock and hypotension. VI. Relationship between left and right ventricular function. Fournal of Clinical Investigation, 48, 2008.

Hutter, A. M., and Moss, A. J. (1968). Value of serial central venous oxygen saturations in acute myocardial infarction. Circulation, 38, Suppl. VI, ro4.

Loeb, H. S., Gunnar, R. M., Pietras, R. J., and Tobin, J. R. (1969). Relationships between central venous and left ventricular filling pressures prior to and during treatment of shock. American fournal of Cardiology, 23, 125.

Nixon, P. G. F., Ikram, H., and Morton, S. (1967). Cardiogenic shock treated with infusion of dextrose solution. American Heart fournal, 53, 843.

—, Taylor, D. J. E., and Morton, S. D. (I968). Left ventricular diastolic pressure in cardiogenic shock treated by dextrose infusion and adrenaline. Lancet, I, 1230.

Norris, R. M., Brandt, P. W. T., Caughey, D. E., Lee, A. J., and Scott, P. J. (I969). A new coronary prognostic index. Lancet, $\mathbf{I}, 274$.

Scheinman, M. M., Brown, M. A., and Rapaport, E. (1969). Critical assessment of use of central venous oxygen saturation as a mirror of mixed venous $0 x y-$ gen in seriously ill cardiac patients. Circulation, 40, 165 . 